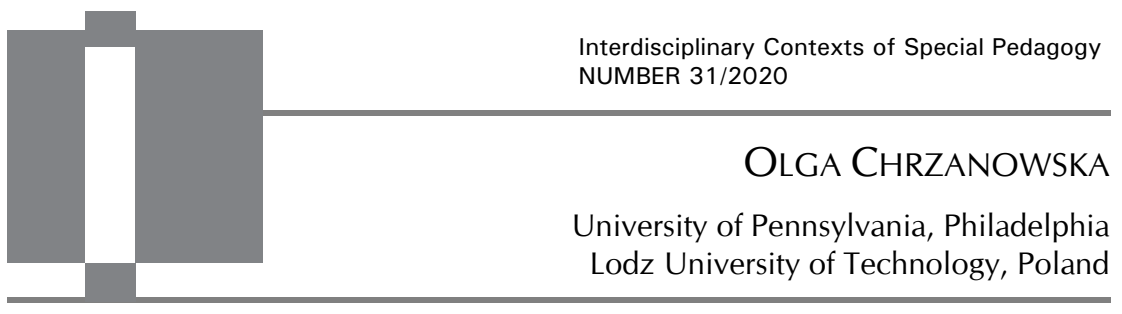

\title{
Universal Design Principles for Older People and Older People with Disabilities During the COVID-19 Pandemic
}

\begin{abstract}
Olga Chrzanowska, Universal Design Principles for Older People and Older People with Disabilities During the COVID-19 Pandemic. Interdisciplinary Contexts of Special Pedagogy, no. 31, Poznań 2020. Pp. 267-286. Adam Mickiewicz University Press. ISSN 2300-391X. e-ISSN 2658-283X. DOI: https://doi.org/10.14746/ikps. 2020.31.12

This study aims to investigate the existing situation of the COVID-19 pandemic from an architectural and urban perspective. The problem analyzed in the study was whether and how universal design could be a useful tool in creating a meaningful response to social repercussions caused by COVID-19. Of the various issues faced today, this study focused on solving the issue of isolation and reducing infections in elderly and elders with disabilities by introducing architectural and spatial modifications. This research showed that all seven universal design principles when introduced at the architectural and urban levels can solve various issues that elderly people face today during the pandemic. It is executed through administrative and institutional push toward better accessibility and inclusion in a build environment that also works for general public interest.
\end{abstract}

KEY WORDS: Universal Design, Isolation, COVID-19, Older People, Disabilities 


\section{Introduction}

This research aims to investigate the existing situation of the COVID-19 pandemic from an architectural and urban perspective. The problem analyzed in the study is whether and how universal design can be a useful design philosophy in creating a meaningful response to social repercussions caused by COVID-19. Of the various groups of people affected by the pandemic, this study focused on elderly people and elders with disabilities. To address this problem, the following questions were posed to understand whether the universal design could help elderly people during the pandemic. Can any of the seven principles of the universal design provide opportunities to solve the isolation of elderly people in senior care facilities? Can the universal design modify public spaces so that the elderlies are less excluded and face lesser risk being infected by COVID-19?

\section{Specific objectives}

The whole study is a form of comparison. It listed seven principles of universal design; then, the chosen social repercussions caused by COVID-19 were presented alongside. It is meant to show whether and how universal design can solve the current social problems that the pandemic poses on elders' lives. The study tested the validity of an existing contemporary architectural and urban design philosophy of universal design in the realm of the existing situation of pandemic. This was intended to provide solutions to the listed problems of social distancing and subsequent isolation of elders. This helped document and interpret the application of universal design philosophy in response to the pandemic. Two parts of the study addressed its specific objectives.

In the first part, universal design was presented as a possible response to the isolation of elders during the COVID-19 pandemic in senior care facilities. The principles of universal design were listed 
and commented on. Subsequently, the study checked which of the seven universal design principles could help elderly people and elders with disabilities feel less isolated during the pandemic. The report considered as an example was the American Institute of Architects' (AIA) Strategies for Senior Living Communities. Finally, the principles of universal design that best address elders in senior living communities during the pandemic are listed.

The second part of the paper consists of the interview with accessibility in architecture expert Stanis Smith. The question posed was whether universal design can help reduce COVID-19 infections among elders in public spaces. Universal design principles and their utterances in architecture were presented and compared to the AIA's report suggestions.

\section{Background and hypothesis}

Aging is an alarming issue across the globe. "People worldwide are living longer. Today, for the first time in history, most people can expect to live in their sixties and beyond. By 2050, the world's population aged 60 years and older is expected to total 2 billion, up from 900 million in 2015". ${ }^{1}$ Thus, research on various aspects of the life of elders is important-their number is growing. When the structure of age in the whole population shifts, it generates consequences in all aspects of life. Eventually, many of us at some point in our lives will experience the aging consequences, maybe even in the form of a disability or will live with someone who becomes disabled.

Even prior to the pandemic, according to World Health Organization (WHO) data "there is, however, little evidence to suggest that older people today are experiencing their later years in better health than their parents. While rates of severe disability have declined in

1 https:/ / www.who.int/news-room/ fact-sheets/detail/ageing-and-health [27.11.2020]. 
high-income countries over the past 30 years, there has been no significant change in mild to moderate disability over the same period. If people can experience these extra years of life in good health and if they live in a supportive environment, their ability to do the things they value will be little different from that of a younger person. If these added years are dominated by declines in physical and mental capacity, the implications for older people and for society are more negative" ${ }^{2}$

The issue studied here was the isolation of elders because of health risks due to COVID-19. As of October 2020, many countries around the world recommended seniors to stay at home and limit their exposure to other people who could possibly be infected. These recommendations and restrictions differed statewide, and they changed in time according to the number of COVID-19 cases and casualties.

The restrictions and recommendations imposed were a form of structural isolation, which means it is an objective limitation of faceto-face social contact. "Social isolation (the objective state of having few social relationships or infrequent social contact with others) and loneliness (a subjective feeling of being isolated) are serious yet underappreciated public health risks that affect a significant portion of the older adult population". 3 "People who are 50 years of age or older are more likely to experience many of the risk factors that can cause or exacerbate social isolation or loneliness, such as living alone, the loss of family or friends, chronic illness, and sensory impairments". 4

2 https://www.who.int/news-room/fact-sheets/detail/ageing-and-health

3 Social Isolation and Loneliness in Older Adults: Opportunities for the Health Care System, "National Academies of Sciences, Engineering, and Medicine. Consensus Study Report", The National Academies Press, Washington, DC 2020, p. 1, https:/ / www.nap.edu/catalog/25663/social-isolation-and-loneliness-in-older-adults-oppor tunities-for-the [27.11.2020].

4 Social Isolation and Loneliness in Older Adults: Opportunities for the Health Care System, "National Academies of Sciences, Engineering, and Medicine. Consensus Study Report", The National Academies Press, Washington, DC 2020, p. 1, https:// www.nap.edu/catalog/25663/social-isolation-and-loneliness-in-older-adults-oppor tunities-for-the [27.11.2020]. 
"Research indicates that social isolation and loneliness increase the risk of mental disorders, but less is known about the distinct contributions of different aspects of isolation".5 "Social network structure and function are strongly intertwined with anxiety and depression symptoms in the general population of older adults. Public health initiatives could reduce perceived isolation by facilitating social network integration and participation in community activities, thereby protecting against the development of affective disorders" ${ }^{6}$

Due to the COVID-19 pandemic, there were restrictions on movement, sheltering in place orders, and other legal and advisory rules that imposed isolation on society. One of the features of isolation is less frequent social interaction. Less frequent social interaction is linked with social disconnectedness, perceived isolation, depression, and anxiety. ${ }^{7}$ Therefore, in the reports of $\mathrm{WHO}^{8}$ and $\mathrm{AIA}^{9}$

5 Z.I. Santini, P.E. Jose, E.Y. Cornwell, A. Koyanagi, L. Nielsen, C. Hinrichsen, C. Meilstrup, K.R. Madsen, V. Koushede, Social disconnectedness, perceived isolation, and symptoms of depression and anxiety among older Americans (NSHAP): a longitudinal mediation analysis, "Lancet Public Health", Elsevier, London 2020, pp. 62-70, 27.11.2020.

${ }^{6}$ Z.I. Santini, P.E. Jose, E.Y. Cornwell, A. Koyanagi, L. Nielsen, C. Hinrichsen, C. Meilstrup, K.R. Madsen, V. Koushede, Social disconnectedness, perceived isolation, and symptoms of depression and anxiety among older Americans (NSHAP): a longitudinal mediation analysis, "Lancet Public Health", Elsevier, London 2020, pp. 62-70, 27.11.2020.

7 Z.I. Santini, P.E. Jose, E.Y. Cornwell, A. Koyanagi, L. Nielsen, C. Hinrichsen, C. Meilstrup, K.R. Madsen, V. Koushede, Social disconnectedness, perceived isolation, and symptoms of depression and anxiety among older Americans (NSHAP): a longitudinal mediation analysis, "Lancet Public Health", Elsevier, London 2020, pp. 62-70, 27.11.2020.

${ }^{8}$ Guidance on Covid-19 for the care of older people and people living in long-term care facilities, other non-acute care facilities and home care updated 23 July 2020, "Institutional Repository for Information Sharing", World Health Organization. Regional Office for the Western Pacific, Manila 2020, https://iris.wpro.who.int/bitstream/handle/ 10665.1/14500/COVID-19-emergency-guidance-ageing-eng.pdf [27.11.2020].

${ }^{9}$ Strategies for Senior Living Communities, The American Institute of Architects, Washington DC 2020, http://content.aia.org/sites/default/files/2020-06/AIA_CO VID-19-Building-Type-Report-Senior-Living_2020-0629.pdf [27.11.2020]. 
on the COVID-19 response in relation to older adults, two major topics were taken into consideration: reducing infections and solving the issue of isolation. In both cases, the organizational recommendations were based on various types of actions. There were suggestions for creating a supportive environment for introducing elders to high-tech equipment for connection, and there were ideas on reshaping activities and creating new forms of social intervention. There were also architectural and design suggestions.

One of the things WHO suggested in their report was to "avoid stereotyping older people as frail and vulnerable". ${ }^{10}$ Individual needs of elders and elders with disabilities are important to take into consideration and generalizing is working against that. Stanis Smith of the Rick Hansen Foundation certifying accessibility in the build environment, suggested the ability to navigate one's environment and dealing with basic needs in the most dignified way possible and needing the least amount of assistance was a fundamental objective of universal design. The universal design idea relies on concrete changes in the environment, not an ad hoc social intervention. Creating an age- and disability-friendly physical environment is an important step in both preparing for future crises and dealing with the current one. This study focuses on the architectural and urban aspects of physical space. Identifying the strain of architecture that could help in this pandemic is considered a vital part in creating a multi-sector response to the crisis that we face. The hypothesis tested in this study was that the more widespread implementation of universal design in architecture and urban planning can solve for the problem of isolation of the elderly caused by the COVID-19 pandemic.

${ }^{10}$ Guidance on Covid-19 for the care of older people and people living in long-term care facilities, other non-acute care facilities and home care updated 23 July 2020, "Institutional Repository for Information Sharing", World Health Organization. Regional Office for the Western Pacific, Manila 2020, https://iris.wpro.who.int/bitstream/handle/ 10665.1/14500/COVID-19-emergency-guidance-ageing-eng.pdf [27.11.2020]. 


\section{Methods and Data}

In this study, qualitative research methods were used. The first was a documentative research with a focus on literature review and policy research aimed at defining current responses to the COVID19 pandemic. Second, this study presents literal quotations from expert interviews showing the technical, process, and interpretative knowledge of the subject of universal design in relation to the pandemic. "Especially in the exploratory phase of a project, interviewing experts is regarded as more efficient and concentrated method of generating data than, for instance, participatory observation" ${ }^{11}$

The reason for choosing these methods was the scope of the research. COVID-19 as a novel problem has generated, to date, only limited literature concerning its topic. Even less literature addressed the rapid responses to the social crisis we face today as the pandemic unfolds. As of October 2020, no monograph on the social consequences of this pandemic in the field of architecture has yet been written. The rapid responses in the form of journal articles and institutional reports were being prepared and implemented just as this paper was being written. The key gray literature material chosen for this study was the American Institute of Architects' (AIA) Strategies for Senior Living Communities.

Therefore, this study used gray literature produced by organizations concerning governance. It "typically includes both policy documents of a traditional form and reports on research done in relation to the issue. The policy documents outline rationales and the present proposal. They can be regarded as traditional primary sources" ${ }^{12}$ Additionally, in this situation, access to knowledgeable experts in the field of universal design in architecture and a chair of

11 A. Bogner, B. Littig, W. Menz, Generating Qualitative Data with Experts and Elite, "The SAGE Handbook of Qualitative Data Collection", SAGE Publications, New York 2018, p. 655.

12 D. Byrne, Reviewing the Literature: What Is Grey Literature?, "Project Planner, SAGE Research Methods", SAGE Publishing, New York 2017. 
organization that provides assessment for accessible sites and buildings is a vital part of the research. The expert chosen for this study was the architect Stanis Smith, a Board member of the Rick Hansen Foundation (RHF), which certifies accessibility of buildings and sites and provides other forms of engagement and innovative solutions for people with disabilities. The Foundation established in 1988 "is a registered Canadian charity (...) that creates and delivers innovative solutions that lead to a global movement to remove barriers and liberate the potential of people with disabilities" ${ }^{13}$

Stanis Smith is a consultant and advisor for various initiatives, one of them being a universal design. Apart from that, Stanis Smith has over 35 years of experience as a corporate executive and architect. He was a leader at Stantec, an international professional services company in the design and consulting industry, including Executive VP for Stantec's Buildings Group, leader of Stantec's Airport Group, and Executive VP for Creativity and Innovation. His expertise in innovation, design, and accessibility as well as leadership, experience in architecture, and current position in RHF made him suitable for providing technical, process, and interpretative knowledge about universal design in relation to the COVID-19 pandemic.

The interview was conducted on November 4th, 2020, at Vancouver, British Columbia, Canada through zoom call with video and audio recording. The interview format was an online face-toface conversation structured around questions that the interviewee got earlier. The length of the interview was about 45 minutes, and the interviewee provided written brief answers to the questions and included additional material in the form of a slide showing presentation and links to additional reports and documents. The audio of an interview was transcribed by an interviewer and later reviewed by an interviewee to supplement the inaudible parts, edits in grammar, and clarification of meaning.

13 https:/ / www.rickhansen.com/about-us/faq [27.11.2020]. 


\section{Results}

\section{Seven Principles of Universal Design and Recommendations from AIA's Report}

United Nations (UN) in their Convention on the Rights of Persons with Disabilities presented the definition of universal design as follows "'Universal design' means the design of products, environments, programmes and services to be usable by all people, to the greatest extent possible, without the need for adaptation or specialized design. 'Universal design' shall not exclude assistive devices for particular groups of persons with disabilities where this is needed". ${ }^{14}$ Then, the UN Convention in Article 3 of General principles stated "The principles of the present Convention shall be: (a) Respect for inherent dignity, individual autonomy including the freedom to make one's own choices, and independence of persons; (b) Non-discrimination; (c) Full and effective participation and inclusion in society; (d) Respect for difference and acceptance of persons with disabilities as part of human diversity and humanity; (e) Equality of opportunity; (...)"..15

Architect Ronald Lawrence Mace pioneered the universal design concept by helping develop an accessible building code in North Carolina in 1973. His history was entwined with one of the previous epidemics that occurred in the twentieth century. He happened to be a polio victim at the age of nine and used a wheelchair since. ${ }^{16}$ Mace also created the Center for Accessible Housing (later

${ }^{14}$ Convention on the Rights of Persons with Disabilities and Optional Protocol, United Nations, New York 2006, p. 4, https://www.un.org/disabilities/documents/con vention/convoptprot-e.pdf [27.11.2020].

15 Convention on the Rights of Persons with Disabilities and Optional Protocol, United Nations, New York 2006, p. 5, https://www.un.org/disabilities/documents/con vention/convoptprot-e.pdf [27.11.2020].

${ }^{16}$ W. Saxon, R. L. Mace, Designer of Buildings Accessible to All, "New York Times", New York 13 July 1998, https:// www.nytimes.com/1998/07/13/us/ronald-l-mace58-designer-of-buildings-accessible-to-all.html [27.11.2020]. 
Center for Universal Design in North Carolina State) and was a president of Barrier Free Environments Inc., a consulting company that offered help in making buildings accessible. ${ }^{17}$ The universal design principles mentioned earlier are a set of seven basic rules listed by the Center for Universal Design (CUD) at North Carolina State University in 1997. These rules were formulated by Bettye Rose Cornell, Mike Jones, Ronald Mace, Jim Mueller, Abir Mullick, Elaine Ostroff, Jon Stanford, Ed Steinfeld, Molly Story, and Gregg Vanderheiden. Seven principles address the architectural and urban environment as well as other forms of interaction with designed entities. Here are the principles with examples provided by Sheryl Burgstahler.

1. "Equitable use. The design is useful and marketable to people with diverse abilities. For example, a website that is designed to be accessible to everyone, including people who are blind and use screen reader technology, employs this principle.

2. Flexibility in use. The design accommodates a wide range of individual preferences and abilities. An example is a museum that allows visitors to choose to read or listen to the description of the contents of a display case.

3. Simple and intuitive. Use of the design is easy to understand, regardless of the user's experience, knowledge, language skills, or current concentration level. Science lab equipment with clear and intuitive control buttons is an example of an application of this principle.

4. Perceptible information. The design communicates necessary information effectively to the user, regardless of ambient conditions or the user's sensory abilities. An example of this principle is captioned television programming projected in a noisy sports bar.

5. Tolerance for error. The design minimizes hazards and adverse consequences of accidental or unintended actions. An ex-

17 W. Saxon, R. L. Mace, Designer of Buildings Accessible to All, "New York Times", New York 13 July, 1998, https://www.nytimes.com/1998/07/13/us/ ronald-1-mace-58-designer-of-buildings-accessible-to-all.html [27.11.2020]. 
ample of a product applying this principle is software applications that provide guidance when the user makes an inappropriate selection.

6. Low physical effort. The design can be used efficiently, comfortably, and with minimum fatigue. Doors that open automatically for people with a wide variety of physical characteristics demonstrate the application of this principle.

7. Size and space for approach and use. Appropriate size and space are provided for approach, reach, manipulation, and use regardless of the user's body size, posture, or mobility. A flexible work area designed for use by employees who are left- or right-handed and have a variety of other physical characteristics and abilities is an example of applying this principle" ${ }^{18}$

The report of the American Institute of Architects' (AIA) Strategies for Senior Living Communities was one of the latest publications prepared by the AIA's Design For Aging Knowledge Community. It was part of the AIA's COVID-19 emerging research and public health data. The Design For Aging (DFA) mission is "to foster design innovation and disseminate knowledge necessary to enhance the built environment and quality of life for an aging society. This includes relevant research on characteristics, planning, and costs associated with innovative design for aging. In addition, DFA provides outcome data on the value of these design solutions and environments". ${ }^{19}$

In the Strategies for Senior Living Communities, the authors emphasized the hazards of isolation of elders. "Long periods of isolation among residents sheltering-in-place, in a restricted access area, or deprived of something as small as having their hand held or the embrace of a grandchild can negatively impact mental health and,

18 S. Burgstahler, Universal Design: Process, Principles, and Applications, "DO-IT (Disabilities, Opportunities, Internetworking, and Technology)", University of Washington, Seattle 2004, http://washington.edu/doit/universal-design-processprinciples-and-applications [27.11.2020].

19 https:/ / network.aia.org/communities/community-home?communitykey=a 83686b6-4432-4ac2-a3a1-b91a6808a014\&tab=groupdetails [27.11.2020]. 
by extension, physical well-being. Reduced access to meaningful activities that provide social connection, personal fulfillment, and exercise can degrade one's physical, emotional, and mental well-being". ${ }^{20}$

The universal design's logic focuses on providing access to include all people in social life despite their various changing needs. Accessibility is one of the main issues addressed by universal design. It is also a problem that people with disabilities and people with disabilities because of old age face in their everyday lives. In Eric Emerson's research conducted in August 2020 on the loneliness of working adults with disability ${ }^{21}$, there was an insight into this problem. The study's aim was "for a nationally representative sample of adults (age 16-64) with/without disability, to examine exposure to three indicators of low social connectedness (loneliness, low perceived social support, social isolation), and to evaluate the association between low social connectedness and wellbeing. To test whether disability status moderated the relationship between low social connectedness and wellbeing".22 The study showed that "people with disabilities experienced loneliness, low perceived social support, and social isolation at significantly higher rates than people without disability. Effect sizes were significantly greater for loneliness". ${ }^{23}$

20 Strategies for Senior Living Communities, The American Institute of Architects, Washington DC 2020, http://content.aia.org/sites/default/files/2020-06/AIA_CO VID-19-Building-Type-Report-Senior-Living_2020-0629.pdf [27.11.2020].

${ }^{21}$ E. Emerson, N. Fortune, G. Llewellyn, R. Stancliffe, Loneliness, Social Support, Social Isolation and Wellbeing Among Working Age Adults with and Without Disability: Cross Sectional Study, "Disability \& Health Journal", American Association on Health and Disability, Rockville, MD August 2020.

22 E. Emerson, N. Fortune, G. Llewellyn, R. Stancliffe, Loneliness, Social Support, Social Isolation and Wellbeing Among Working Age Adults with and Without Disability: Cross Sectional Study, "Disability \& Health Journal", American Association on Health and Disability, Rockville, MD August 2020.

${ }^{23}$ E. Emerson, N. Fortune, G. Llewellyn, R. Stancliffe, Loneliness, Social Support, Social Isolation and Wellbeing Among Working Age Adults with and Without Disability: Cross Sectional Study, "Disability \& Health Journal", American Association on Health and Disability, Rockville, MD August 2020. 
The role of the design of the environment in social inclusion is not overlooked. "Design that does not cater for a diverse range of ages, abilities and cultures restricts people's access to, and use of, domestic or public premises. Universal design is an approach that acknowledges diversity of populations and encourages designers to create objects and places that are usable by the greatest majority of users". ${ }^{24}$ In the Preamble of the UN Convention on the Rights of Persons with Disabilities there as point made on (v) Recognizing the importance of accessibility to the physical, social, economic and cultural environment, to health and education and to information and communication, in enabling persons with disabilities to fully enjoy all human rights and fundamental freedoms (...).

Inclusion in build environment is also stated in Article 9 on Accessibility " 1 . To enable persons with disabilities to live independently and participate fully in all aspects of life, States Parties shall take appropriate measures to ensure to persons with disabilities access, on an equal basis with others, to the physical environment, to transportation, to information and communications, including information and communications technologies and systems, and to other facilities and services open or provided to the public, both in urban and in rural areas. These measures, which shall include the identification and elimination of obstacles and barriers to accessibility, shall apply to, inter alia: (a) Buildings, roads, transportation and other indoor and outdoor facilities, including schools, housing, medical facilities and workplaces; (...)" ${ }^{25}$

The AIA's report provided possible strategies to reduce the additional isolation that is now a consequence of COVID-19 infection in elderly care facilities. Many of the suggestions were of use in

24 V. Watchorn, H. Larkin, D. Hitch, S. Ang, Promoting Participation Through the Universal Design of Built Environments: Making It Happen, "Journal of Social Inclusion", School of Human Services and Social Work, Griffith University p. 87, Nathan, QLD 2014.

25 Convention on the Rights of Persons with Disabilities and Optional Protocol, United Nations, New York 2006, p.4, https://www.un.org/disabilities/documents/con vention/convoptprot-e.pdf [27.11.2020]. 
housing and public spaces. Among others, there were advices to "Find ways for residents to connect with neighbors, visitors, family, and friends to help them maintain vital emotional and social bonds. Arrange virtual visits on laptops or smartphones. Incorporate testing protocols for prospective visitors and arrange small gatherings in outdoor areas like gazebos or where there are benches. Provide alternatives for meaningful activities that prevent risk. When the communal risk is low enough, seating can be arranged so smallgroup conversations can be heard through masks when physically distanced. Accommodations for the hearing impaired should be made". 26

The report also showed examples of tactics to solve isolation and reduce infections in senior living facilities. They suggested small fixes within the way people use existing sites. They mentioned to "Open assembly areas with restricted occupant loads. Provide more exterior activities in limited groups. Control access to areas to limited small groups assigned to each area. Limit areas of public visitation. Add handwashing stations. Provide exterior entrance to some units to limit public access to all residents. Provide in-room dining area for family visitation. Open up wing entrances to limit location of outside visitors. Increase building pets" ${ }^{27}$

Several of the AIA's recommended strategies for solving isolation of elderly could be achieved at a site when the first, second, and seventh universal design principles were in place, that is, equitable use, flexibility in use, and size and space for approach and use. Arranging, re-arranging, and accommodating the existing space is an example of flexibility and equity of use in the design. This can relate to the changes in the layout of the space itself, as spaces may need to be enlarged, joined, moved, or divided to ensure a safer meeting

26 Strategies for Senior Living Communities, The American Institute of Architects, Washington DC 2020, http://content.aia.org/sites/default/files/2020-06/AIA_CO VID-19-Building-Type-Report-Senior-Living_2020-0629.pdf [27.11.2020].

27 Strategies for Senior Living Communities, The American Institute of Architects, Washington DC 2020, http://content.aia.org/sites/default/files/2020-06/AIA_CO VID-19-Building-Type-Report-Senior-Living_2020-0629.pdf [27.11.2020]. 
environment. Equity is reflected in adaptations for specific needs, such as in the example of physically distanced meetings in masks by adding accommodations for the hearing impaired. Changes introduced to the functioning of the space, such as entering and leaving rooms and modifying other aspects of one's behavior in rooms, require both flexibility of use and size and space for approach and use from the design itself.

There were three universal design principles that could help solve the isolation of elders and elders with disabilities in elderly care facilities, but what about other principles of universal design? Are they of use in sites other than senior care facilities and other scenarios where people enter the public space during the COVID-19 pandemic?

\section{Universal design features for reducing infections}

Reducing infections among seniors in elderly care facilities was also considered possible by architectural and design interventions according to the AIA's report. Some of the solutions were closely bounded by the universal design philosophy. For reducing infections, suggestions were "If possible, replace water fountains with touchless water dispensers" .28 When entering and leaving common areas, solutions, including sliding doors activated by elbow were encouraged. Grab-and-go items, touchless payment systems, and reducing shared exercise equipment were suggested as ways to control the transmission of COVID-19 by limitation of surface touching.

Touchless solutions for low physical effort in universal design could work for better hygiene in various public spaces. Low physical effort is sixth of the seven universal design principles. The idea

28 Strategies for Senior Living Communities, The American Institute of Architects, Washington DC 2020, http://content.aia.org/sites/default/files/2020-06/AIA_CO VID-19-Building-Type-Report-Senior-Living_2020-0629.pdf [27.11.2020]. 
of "touchless travel experience" 29 at the airports also corresponds to that issue. Stanis Smith explained it as a concept where from the moment you get out of your vehicle and go through the front door, all the way through the airport to your aircraft at the gate. everything will be touchless. With the current pandemic, there is much more emphasis on this concept because there is currently a health reason to do it not just a convenience reason or universal design reason. So, I believe that the touchless travel experience will become an expectation, touchless door openers, touchless washroom fixtures, touchless passport readers, etc. ${ }^{30}$

When orientation in and usage of public space is easier, quicker, and in need of less assistance, there are health benefits to it. The more convenient the signage and use of space, the less time inside is required and less confusion is caused. Airports are among the few examples of intricate, very well thought through public spaces, solving issues of safety, undisturbed flow, and heavy load of people. Stanis Smith lists options for solutions originating from universal design. Then there are hearing loops, visual displays, and high contrast tactile signs for people with auditory challenges. Many other things in the airport are quite subtle, for example, using floor patterns and lighting as wayfinding devices. ${ }^{31}$

AIA's report encouraged reducing infections in entryways, lobbies, circulation spaces, and restrooms as follows. "Designate a separate entrance and holding area for deliveries. Provide signage and floor markings throughout the building to encourage physical separation of residents, staff, and visitors. Separate seating and other work surfaces a minimum of six feet or further, depending on the activity. Install transparent sneeze guards in the reception area". Signage and floor marking solutions are intended to limit the error of residents, delivery, staff, and visitors running into each other

29 Interview with Stanis Smith, Universal Design in the Era of Covid-19, Vancouver BC, 4 November 2020.

30 Interview with Stanis Smith, Universal Design in the Era of Covid-19, Vancouver BC, 4 November 2020.

31 Interview with Stanis Smith, Universal Design in the Era of Covid-19, Vancouver BC, 4 November 2020. 
while wandering around the site. Tolerance for error is the fifth universal design rule. Transparent sneeze guards protect from immediate and direct exposition on uncovered sneezes or coughs of others.

For dining areas, AIA's recommends "Implementing floor markings to locate tables. Reducing the amount of equipment to provide more spacing. Menu on chalkboard on the wall and/or disposable menu and braille menu; alternatively, provide menu on the reservation system. TV screens for facility safety reminders, program schedule, and entertainment. Provide regular seating for couples and shared households, if desired. Tables reserved for those not in the same household should provide additional distancing". These solutions utilize the third and fourth principles of universal design of simple and intuitive use and perceptible information. Making navigation through the environment more easy, and therefore, the whole time spent in public or common space more efficient and safer is vital in this pandemic.

This study showed examples where three out of seven principles of universal design can address the isolation of elders in assisted living communities. By implicating the three rules that are equitable use, flexibility in use and size and space for approach and use, the rearrangements and changes of functioning of the spaces, such as those recommended by AIA's report, are more achievable. By certain reorganization of the layout and functioning in the spaces in elderly care facilities, solving the issue of isolation of elders in the COVID-19 pandemic is expected.

Apart from solving the isolation issue, universal design principles when applied in the architectural and urban environments of public spaces could also reduce infections. The third and fourth principles of simple and intuitive use and perceptible information are making navigation through to the environment more easily, and therefore, the whole time spent in public space is more efficient. The fifth and sixth rule of tolerance for error and low physical effort could minimize accidents, fatigue, and the need for help and support on the site. Touchless travel experience in airports or public 
transportation systems could reduce contact with surfaces and the need for additional assistance, thereby increasing hygiene.

This makes all seven universal design principles that are implementation of universal design in architecture and urban planning capable of solving the problem of isolation of elderly or for reducing their chances of being infected during the COVID-19 pandemic. There are various examples in existing sites built in the spirit of universal design, and in recommendations from top institutions, showing that the philosophy of universal design can create a meaningful response to social repercussions caused by COVID-19.

\section{Discussion}

Widespread implementation of universal design can be highly beneficial to society, even without a pandemic. Elderlies with preexisting health conditions and older people with disabilities have already had limited access to public spaces. They were already isolated when accessible sites were not provided or were not in proximity. When the site was not easy to navigate through and many of its functions could not be approached by the elderlies, it excluded them from social life in that area. It has become an alarming issue in aging societies, making numerous people each year in need of accessibility improvements. As Stanis Smith points out, I think universal design is more of a philosophical and moral imperative, for us to find ways that everybody can contribute to society. If there are ways to make the built environment more accessible, that obviously helps people who have disabilities, but it also helps society as a whole. ${ }^{32}$

In the Preamble of the UN Convention on the Rights of Persons with Disabilities, there were points made on "(..) (m) Recognizing the valued existing and potential contributions made by persons with disabilities to the overall well-being and diversity of their

32 Interview with Stanis Smith, Universal Design in the Era of Covid-19, Vancouver BC, 4 November 2020. 
communities, and that the promotion of the full enjoyment by persons with disabilities of their human rights and fundamental freedoms and of full participation by persons with disabilities will result in their enhanced sense of belonging and in significant advances in the human, social, and economic development of society and the eradication of poverty, (n) Recognizing the importance for persons with disabilities of their individual autonomy and independence, including the freedom to make their own choices, $(\ldots)^{33}$

The current pandemic makes isolation of elders even more severe as another factor was introduced-risk of being infected with COVID-19. The rate of this additional isolation and its consequences are not yet known. Activities were disrupted in both public spaces and semi-public or semi-private spaces in everyday environments, where interactions between people frequently occurred. Many of the sites remain or are temporarily closed like museums and theaters. However, social interactions also take place in markets, common areas of stairwells and corridors of housing units, public squares, or senior care facilities, most of which cannot be closed. Research in the architectural and urban planning sector is highly needed, so the physical environment can address life in the realm of this and other upcoming crises.

\section{References}

Bogner A., Littig B., Menz W., Generating Qualitative Data with Experts and Elite, "The SAGE Handbook of Qualitative Data Collection" 2018, p. 655.

Burgstahler S., Universal Design: Process, Principles, and Applications, "DO-IT (Disabilities, Opportunities, Internetworking, and Technology)" 2004, https://www. washington.edu/doit/universal-design-process-principles-and-applications, 27.11.2020.

Byrne D., Reviewing the Literature: What Is Grey Literature?, „Project Planner SAGE Research Methods", SAGE 2017.

33 Convention on the Rights of Persons with Disabilities and Optional Protocol, United Nations, New York 2006, p. 4, https://www.un.org/disabilities/documents/con vention/convoptprot-e.pdf [27.11.2020]. 
Chrzanowska O., Interview with Stanis Smith, Universal Design in the Era of Covid-19, Vancouver British Columbia, November 4, 2020.

Emerson E., Fortune N., Llewellyn G., Stancliffe R., Loneliness, social support, social isolation and wellbeing among working age adults with and without disability: Cross sectional Study, Disability Health Journal August 2020.

National Academies of Sciences, Engineering, and Medicine, Social Isolation and Loneliness in Older Adults: Opportunities for the Health Care System, "National Academies of Sciences, Engineering, and Medicine. Consensus Study Report", Washington, DC: The National Academies Press 2020, p. 1, https://www.nap. edu/catalog/25663/social-isolation-and-loneliness-in-older-adults-opportuni ties-for-the, 27.11.2020.

Santini Z.I., Jose P.E., Cornwell E.Y., Koyanagi A., Nielsen L., Hinrichsen C., Meilstrup C., Madsen K.R., Koushede V., Social Disconnectedness, Perceived Isolation, and Symptoms of Depression and Anxiety Among Older Americans (NSHAP): a Longitudinal Mediation Analysis, "Lancet Public Health”, The Lancet 2020, p. 62-70.

Saxon W., Ronald L. Mace, 58, Designer Of Buildings Accessible to All, New York Times July 13 1998, Section B, p. 9, https://www.nytimes.com/1998/07/13/us/ro nald-1-mace-58-designer-of-buildings-accessible-to-all.html, 03.12.2020.

The American Institute of Architects, Strategies for Senior Living Communities, Washington DC 2020, p.6, http://content.aia.org/sites/default/files/2020-06/AIA_ COVID-19-Building-Type-Report-Senior-Living_2020-0629.pdf, 27.11.2020.

United Nations, Convention on the Rights of Persons with Disabilities and Optional Protocol, United Nations New York 2006, p. 9, https://www.un.org/disabilities/ documents/convention/convoptprot-e.pdf, 27.11.2020.

World Health Organization, Guidance on Covid-19 for the care of older people and people living in long-term care facilities, other non-acute care facilities and home care updated 23 July 2020, World Health Organization 2020, https://iris.wpro.who.int/bit stream/handle/10665.1/14500/COVID-19-emergency-guidance-ageing-eng.pdf, 27.11.2020.

https:// network.aia.org/communities/community-home?communitykey=a83686b 6-4432-4ac2-a3a1-b91a6808a014\&tab=groupdetails, 27.11.2020.

https://www.rickhansen.com/about-us/faq, 27.11.2020. 